\title{
Influences of the PCM Microcapsules on Thermal Properties of the Garment
}

\author{
LI Fengzhi ${ }^{1, a}$, REN Penghao ${ }^{2, b}$ \\ ${ }^{1,2}$ College of Aerospace Engineering, Nanjing University of Aeronautics and Astronautics, Nanjing, \\ 210016, China \\ ahelifz@nuaa.edu.cn, b863741949@qq.com
}

Keywords: PCM Microcapsules; Thermal Properties; Heat and Moisture Transfer; Model; Simulation

\begin{abstract}
To study the influences of phase change material (PCM) microcapsules in the garment on human thermal responses, a mathematical model is developed. The improved Stolwijk's model is used to simulate human thermo-regulatory process, and the coupled heat and moisture transfer including the moisture sorption /desorption of fibers and effects of the PCM is considered in garment model. Meanwhile, the theoretical predictions were validated by experimental data. Then, the interactions between human body thermal responses and the heat and moisture transfer in clothing are discussed by comparing the prediction results with PCMs and without PCMs. The conclusion shows that the clothing with PCMs microcapsules can delay the human temperature variations, decrease the sweat accumulation rate on the skin surface and heat loss during changing of ambient conditions.
\end{abstract}

\section{Introduction}

In recent years, functional textiles have been developed to enhance textile performances according to the consumers' demand. The textiles with phase change material (PCM) microcapsules possess the ability to absorb and store large amounts of latent heat during the heating process and release this energy during the cooling process. There were many researches to manufacture textiles with PCMs microcapsules and investigate the properties of the clothing with PCMs microcapules by experimental methods[1,2] and theoretical methods[3,4]. However, the above studies mentioned olny dry fabric was discussed. Actually, most of textile fibers are hygroscopic, and this influences thermal comfort significantly. To consider the interaction between the fiber hygroscopicity and phase change of the PCM, Refs. [5,6] developed the mathematical models for the clothing. In order to study the influence of clothing with PCM microcapsules on human thermal responses, this paper develops a new model of heat and moisture transfer in human-clothing with PCM microcapsules-environment system based on Ref. [6] and improved Stolwijk’s human thermo-regulatory model [7].

\section{Mathematical Model}

The controlled system of the Stolwijk's model consists of cylinders of different sizes representing trunk, arms, hands, legs and feet, and the head is represented as a sphere. The cylinders or segments are subdivided into four concentric layers representing the core, muscle, fat, and skin layers. An additional central blood pool representing the large arteries and veins, exchanges heat with all other compartments via the convective heat transfer occurring with the blood flow to each layer. The heat balance equations for each layer and blood can be written. For example, the heat balance equation for the skin layer can be written as:

$$
C(i, 4) \frac{d T(i, 4)}{d t}=Q(i, 4)-B(i, 4)+D(i, 3)-E(i, 4)-Q_{t}(i, 4)
$$

where, $i$ denotes the segment, and 4 and 3 denote the skin and fat layers. $C$ is thermal capacity, $T$ is temperature, $t$ is the time, $Q$ is the metabolic rate, $B$ is the heat loss by blood flow, $D$ is the heat flow 
from $i$-th segment's 3-rd layer by the thermal conduction, $R E S$ is respiration heat loss, $Q_{t}$ is the heat loss by thermal radiation and convection, $E$ is the heat loss by evaporation through the skin surface, it can be determined by the Jones method [8]. The control system is used to determine the thermoregulatory amount, such as sweat, chill and blood flow.

Here, the equations of heat and moisture transfer in clothing with PCMs microcapsules developed in Ref. [6] are used.

$$
\left\{\begin{array}{l}
\frac{\partial\left(\varepsilon_{a} C_{a}\right)}{\partial t}+\varepsilon_{f} \frac{\partial C_{f}}{\partial t}=\frac{\partial}{\partial x}\left(\frac{D_{a} \varepsilon_{a}}{\tau} \frac{\partial C_{a}}{\partial x}\right) \\
C_{v} \frac{\partial T}{\partial t}-\lambda \varepsilon_{f} \frac{\partial C_{f}}{\partial t}=\frac{\partial}{\partial x}\left(k \frac{\partial T}{\partial x}\right)+h_{e} \frac{3 \varepsilon_{m}}{R_{m}}\left\{T_{m}\left(x, R_{m}, t\right)-T\right\} \\
\varepsilon=\varepsilon_{a}+\varepsilon_{m}=1-\varepsilon_{f}
\end{array}\right.
$$

where, $\varepsilon_{a}, \varepsilon_{f}$, and $\varepsilon_{m}$ are the volumetric fractions of air filling inter-fiber space, fibers, and PCMs microcapsules, respectively, $C_{a}$ is the water vapor concentration in the air filling inter-fiber space, $C_{f}$ is the mean water vapor concentration in the fibers of fabric, $D_{a}$ is the diffusion coefficient of water vapor in air, $\tau_{a}$ is the effective tortuosity of water vapor through the fabric, $T$ is the fabric temperature, $c_{v}, \lambda$ and $k$ represent the volumetric heat capacity of the fabric, the heat of sorption of water vapor by fibers, and the thermal conductivity of the fabric, which are functions of the water content of the fibers, respectively. $h_{e}$ is the effective heat transfer coefficient between fabric and PCM. $T_{m}\left(x, R_{m}, t\right)$ is the temperature at the surface of the PCM microcapsule, which is a function of position $x$ of the PCM microcapsule in the fabric and time $t$. The mean water vapor concentration in the fibers of fabric, $C_{f}$ can be obtained by Fick's Law [10]. $T_{m}\left(x, R_{m}, t\right)$ can be obtained by solving the thermal balance equation for the PCM sphere [6]:

$$
\tilde{C} \frac{\partial T_{m}(x, r, t)}{\partial t}=\frac{1}{r^{2}} \frac{\partial}{\partial r}\left(\tilde{k} r^{2} \frac{\partial T_{m}(x, r, t)}{\partial r}\right)
$$

with initial and boundary conditions[6]

To solve the equations, we must list the boundary conditions for human body and clothing. In Ref.[11], we have listed the boundary conditions including the water vapor evaporation, heat conduction and radiation bewteen the skin and clothing, and convection and radiation heat transfer and convection moisture transfer between the clothing and environment. Here we use the same conditions. More detailed information about the boundary conditions can be found in the Ref.[11].

\section{Model Validation}

To validate the model, the following experimental process is simulated. Initially, a person dressed in the T-shirt with PCM microcapsules and cotton trousers sits on a chair in the room at $297.45 \mathrm{~K}$, $55.3 \% \mathrm{RH}$ and $\mathrm{v}=0.1 \mathrm{~m} / \mathrm{s}$ for $20 \mathrm{~min}$. to reach an equilibrium state. Then, the experiment starts, the environment temperature falls. After $21 \mathrm{~min}$., the environment temperature raises for $15 \mathrm{~min}$.. The variations of the environment temperature with time are shown in Fig.1. The temperatures at the skin surface and inner surface of clothing are measured. T-shirt is made of polypropylene and 5\% PCM microcapsules. The phase change temperature range is [304.15, 306.45] $\mathrm{K}$, and peak temperature, Tb $=305.95 \mathrm{~K}$. The radius of the microcapsules is $5 \mu \mathrm{m}$. Total phase change latent is $124 \mathrm{~kJ} / \mathrm{kg}$. Other parameters of clothing can be obtained from Ref. [11]. The comparison of the model predictions and experimental measurements is shown in the Fig. 1, from which, we can see that the theoretical predictions and experimental data have a good agreement. During environment temperature raises, the clothing and skin temperature also increases due to the heating of environment. About 27 min., 
the increase of clothing temperature is delayed due to the heat absorbed by the PCM during the phase change process.
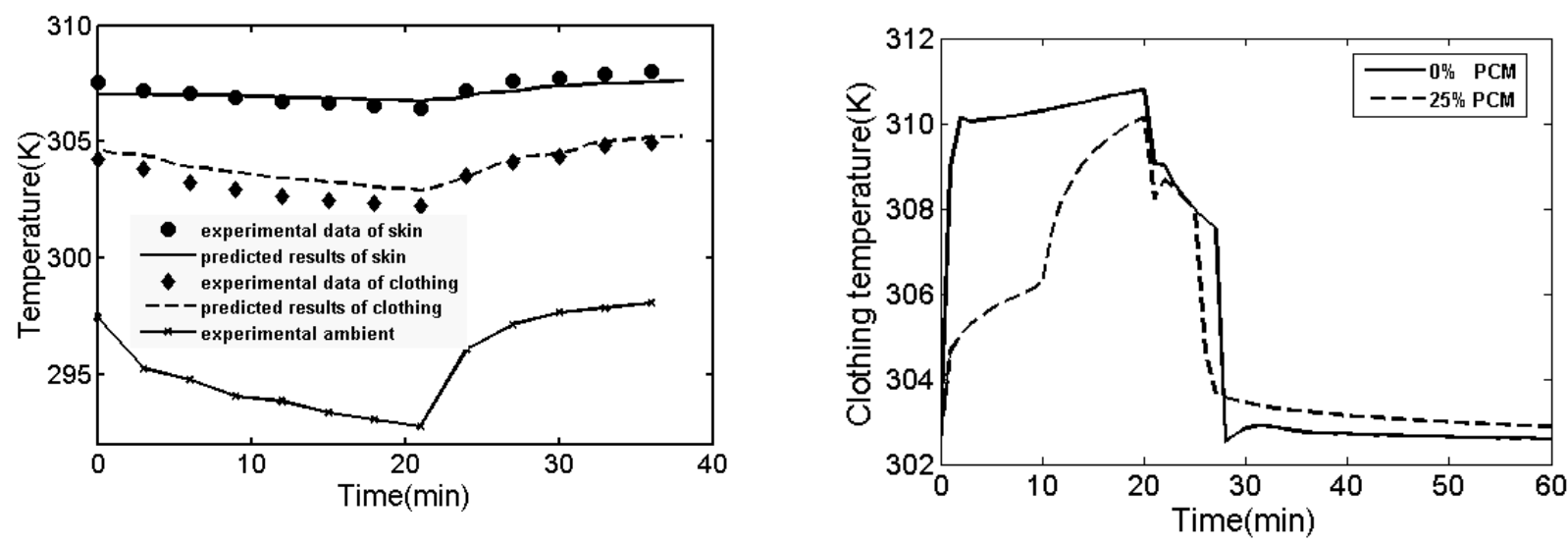

Fig.1 Comparison of model predictions and experimental data

Fig.2 Temperature at inner surface of clothing near trunk

After validating the model, we can use it to investigate the effect of the clothing with or without PCM on responses of human body. The following protocol is simulated. In the first stage, the human body enters a cool room A ( $25 \mathrm{oC}, 40 \% \mathrm{RH}, 0.3 \mathrm{~m} / \mathrm{s})$, wearing the experimental clothing. The clothed human body sits on a chair in room A for 15 minutes to reach equilibrium state. Then, the experiment starts. The subjects move into a hot room B $(360 \mathrm{C}, 80 \% \mathrm{RH}, 0.1 \mathrm{~m} / \mathrm{s})$ and stay in a seated position for 20 minutes. After that they return to room A again and rest for 40 minutes. The T-shirt is made of the same basic materials (polypropylene), but different PCM contents ( $0 \%, 25 \%)$. In the simulation, the clothing thickness is $2.0 \mathrm{~mm}$, and porosity is 0.88 . The physical properties of basic materials can be found in Ref. [11].

Fig. 2 shows the temperature at inner surface of clothing near the trunk. The temperature increase at inner surface of clothing with 25\% PCM is delayed from 0 to $10 \mathrm{~min}$ in high environmental temperature. This is because the PCM is changing from solid to liquid and absorbing heat around the clothing when the PCM gets to its changing temperature. After $10 \mathrm{~min}$, the PCM stops absorbing heat and taking place phase change, the temperature goes on increasing. In the low temperature and humidity environment, the temperature of clothing is decreasing until $21 \mathrm{~min}$. Because of the decrease of the environmental humidity, the evaporation heat from skin to clothing increases suddenly and so the temperature of clothing returns up. After that, the temperature of clothing goes on decreasing in the low environmental temperature. Because the sweat accumulation on truck skin decreases to 0 , the temperature of clothing and the evaporation heat from skin to clothing decreases suddenly around 25 min. Finally the temperature of clothing closes to the equilibrium with the surrounding. The temperature of clothing with $0 \%$ PCM changes similarly without delay.

Fig. 3 shows the temperature variation on the trunk skin surface. The trunk skin temperature of the person dressed in clothing with 25\% PCM is lower and delayed obviously due to the effect of the PCM absorbing heat through melting. In the low temperature environment the temperature of skin returns up for a while due to the effect of the accommodation of blood. Fig. 4 shows the water vapor concentration at clothing inner surface. From 2 to $10 \mathrm{~min}$ in high temperature environment the water vapor concentration at clothing with 25\% PCM is lower than that without PCM due to the effect of the water vapor pressure at skin surface and that in the environment. The water vapor concentration at skin surface is delayed due to PCM influence. After 25 min., in low temperature and humidity environment, the water vapor concentration at inner surface of clothing with 25\% PCM decreases quickly because the sweat accumulation on trunk skin decreases to 0 and water vapor pressure on the skin decreases at this moment. Around $28 \mathrm{~min}$ the water vapor concentration at the inner surface of the clothing without PCM has the similar variation. 


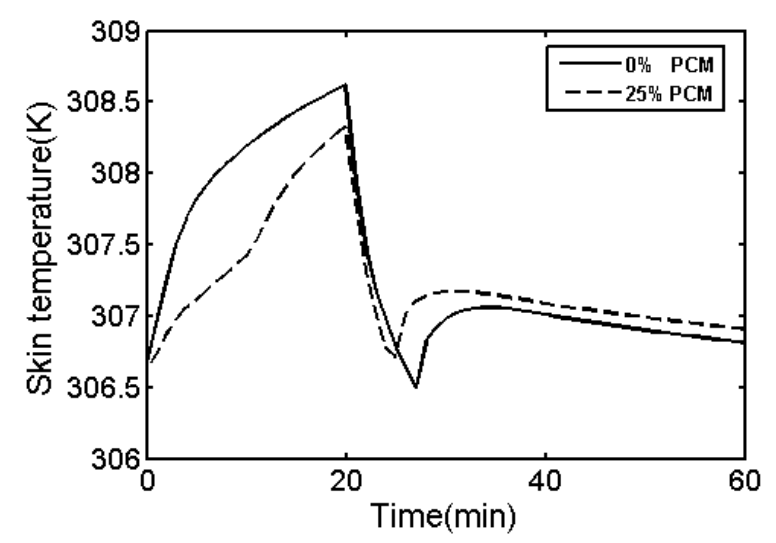

Fig.3 Temperature of trunk skin

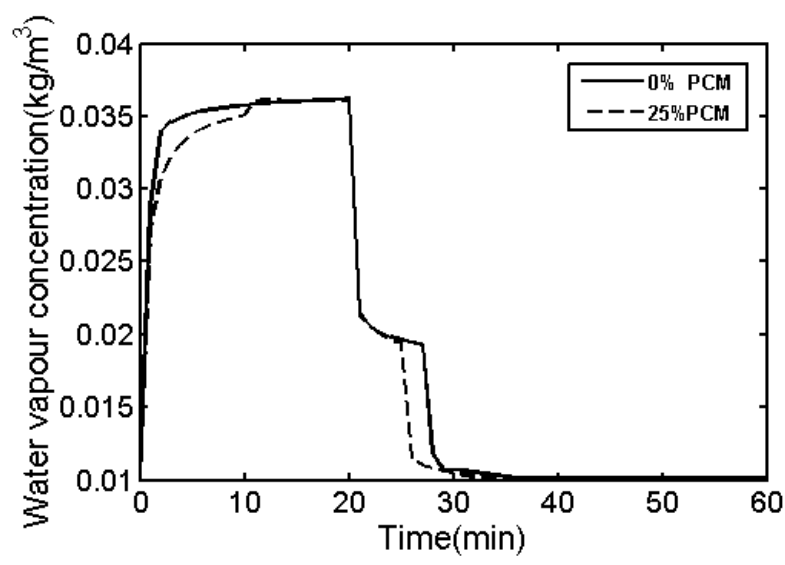

Fig. 4 Water vapor concentration at clothing inner surface

Fig. 5 is the sweat accumulation on trunk skin surface. In high temperature environment human begins sweating and the sweat accumulation increases at skin surface. In low temperature environment the sweat accumulation decreases to 0 . The time of the sweat accumulation on trunk skin surface is shorter when human is on clothing with 25\% PCM. Fig. 6 shows dry heat loss from the trunk skin. Dry heat loss is depended on the temperature difference between skin and clothing. In high temperature environment, dry heat loss is minus because the environment temperature is higher than skin surface temperature and body on clothing with 25\% PCM earns less heat than that on clothing without PCM from clothing. In low temperature environment, dry heat loss is plus because heat transfers from skin to clothing and at most time dry heat loss of body on clothing without PCM is more than that on clothing with 25\% PCM.

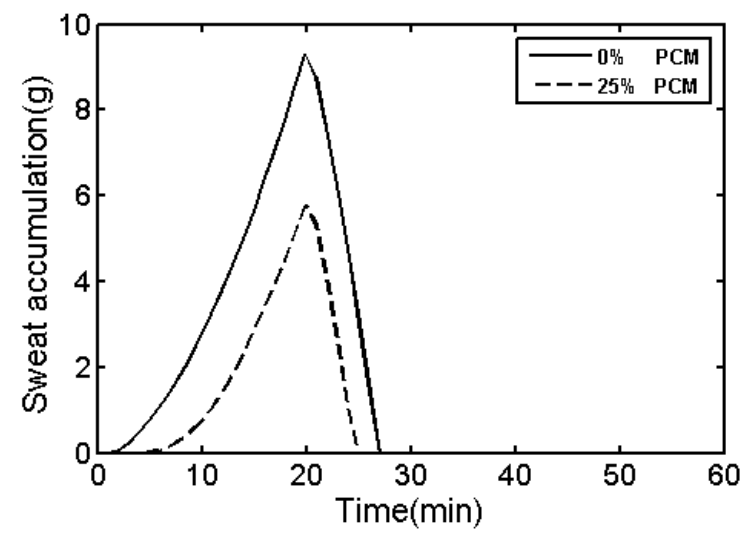

Fig. 5 Sweat accumulation on the trunk skin

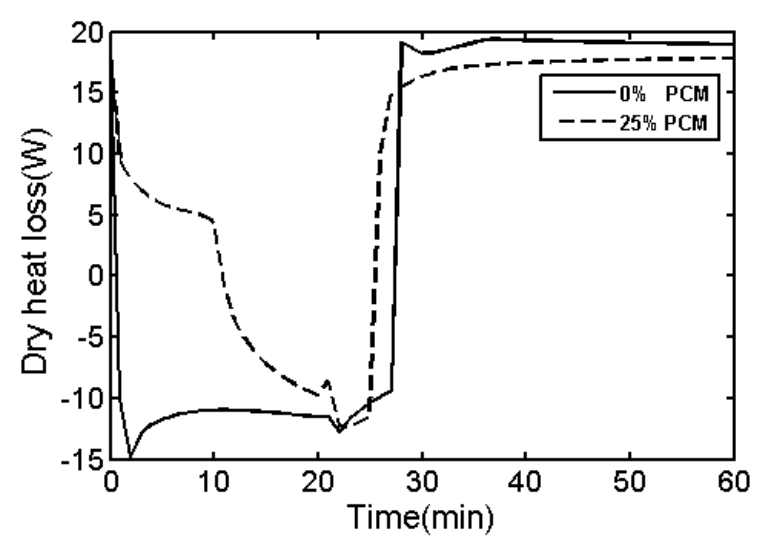

Fig. 6 Dry heat loss of trunk skin

Fig.7 shows evaporative heat loss from trunk skin surface. In high temperature environment evaporative heat loss at trunk skin surface on clothing with 25\% PCM is more than that on clothing without PCM. Evaporative heat loss varies directly as the water vapor pressure difference between skin surface and clothing inner surface. In low humidity environment evaporative heat loss increases quickly because the water vapor pressure at clothing inner surface decreases quickly due to the lower environment humidity after $20 \mathrm{~min}$. The temperature of human decreases as the water vapor pressure at skin surface, so evaporative heat loss decreases. Around 25 min evaporative heat loss at trunk skin surface on clothing with 25\% PCM decreases quickly because the sweat accumulation at skin surface decreases to 0 and the water vapor pressure at skin surface decreases quickly. The clothing without PCM has similar phenomenon around 28 min. Fig. 8 shows heat exchange rate between blood and trunk skin. Because the temperature of blood is higher than that of skin, heat is transferred from blood to skin. In high temperature environment the heat transferred from blood to skin on clothing with $25 \%$ 
PCM is more than that on clothing without PCM, because at most of time the temperature of skin on clothing with 25\% PCM is lower than that on clothing without PCM.

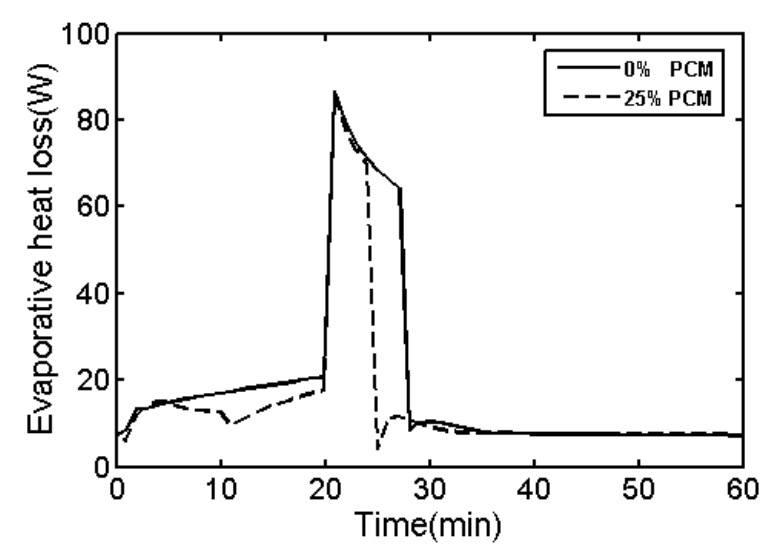

Fig. 7 Evaporative heat loss at trunk skin surface

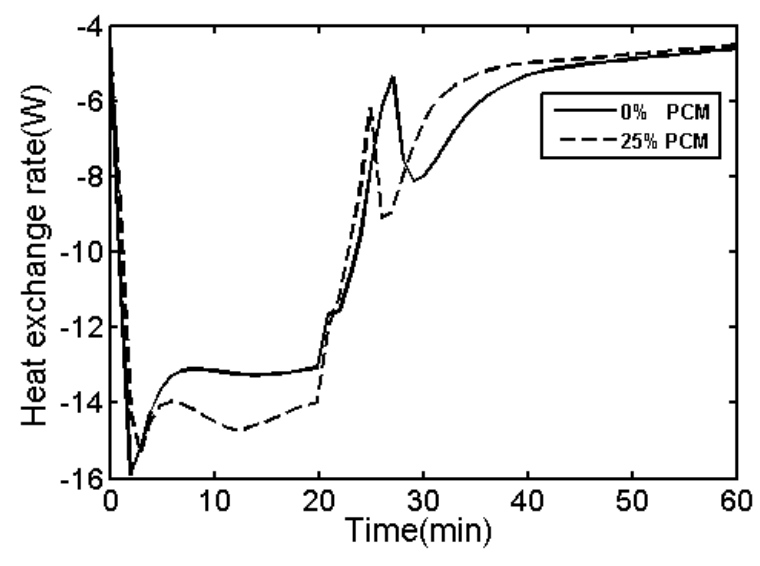

Fig. 8 Heat exchange rate between blood and trunk skin

\section{Conclusion}

This paper discussed the influences of the PCM added in the clothing on heat and moisture in clothed human body by using the new model developed in this paper. Comparing the thermal responses of human body dressed in clothing with PCM and that without PCM, we found that the PCM microcapsules in clothing can delay the raising of clothing temperature, decrease human body temperature and sweat accumulation on the skin surface when human body is in the hot environment, whereas, the PCM can delay temperature decreasing of the clothing and skin when the human body is in the cold environment. The model can provide a tool to analysis and design the thermal function of the clothing with PCM.

\section{Acknowledgment}

This work was supported by the Fundamental Research Founds for Central Universities ( NS2013007).

\section{References}

[1] Y. G. Bryant:HTD, 363/BED-44, ASME,1999

[2] H. Shim: Textile Research J. Vol. 71 (2001), p.495

[3] M. L. Nuckols:Ocean Eng. Vol. 26 (1999), p.547

[4] D. C. Hittle, T. L Andre: ASHRAE Transactions Vol. 108 (2002) , p175

[5] Y. Li and Q. Y. Zhu: Textile Research J. Vol.74(2004), p. 447

[6] F. Z. Li, Y. Li:Model. and Simul. in Mater. Sci. and Eng, Vol. 15(2007), p. 223

[7]J . A. J. Stolwijk, and J. D. Hardy: Handbook of physiology reactions to environment agent. (1977), p. 45

[8] B. W. Jones, Y. Ogawa:ASHRAE Trans. Vol. 98(1992), p. 189

[9] A. Yigit: Int. Comm. Heat and Mass Transfer. Vol. 25(1998), p.969

[10] Y. Li, Z. X. Luo: Textile Res. J. Vol. 69 (1999), p.760

[11] F. Z. Li and Y. Li: Modelling Simul. Mater. Sci. Eng. Vol. 13 (2005), p. 809 